Supporting Information

\title{
Computational Screening of Layered Materials for Multivalent Ion Batteries
}

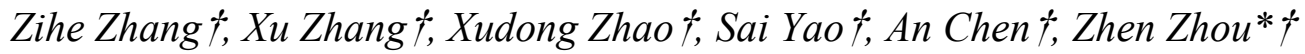

$\dagger$ School of Materials Science and Engineering, Computational Centre for Molecular

Science, Institute of New Energy Material Chemistry, Nankai University, Tianjin

300350, P. R. China. E-mail: zhouzhen@nankai.edu.cn 
Table S1. Remaining compound numbers after each screening step.

\begin{tabular}{ccccccc}
\hline criteria & $\mathrm{Al}$ & $\mathrm{Ca}$ & $\mathrm{Mg}$ & $\mathrm{Li}$ & $\mathrm{Na}$ & $\mathrm{K}$ \\
\hline containing specific metal & 3742 & 4449 & 9818 & 13943 & 4826 & 3954 \\
containing proper element & 2935 & 4140 & 8747 & 13342 & 4354 & 3452 \\
in ICSD & 1387 & 1578 & 946 & 1559 & 2108 & 2364 \\
energy above hull $<0.05 \mathrm{eV} /$ atom & 1188 & 1353 & 776 & 1329 & 1825 & 2008 \\
band gap $<2.5 \mathrm{eV}$ & 615 & 769 & 375 & 634 & 833 & 1083 \\
capacity $>120 \mathrm{mAh} \mathrm{g}{ }^{-1}$ & 581 & 659 & 324 & 412 & 515 & 520 \\
not alloy & 241 & 424 & 138 & 279 & 457 & 480 \\
layered structure & 39 & 100 & 29 & 60 & 81 & 59 \\
\hline
\end{tabular}

The containing proper element means a compound does not contain lanthanide, actinides or noble gas elements. Not alloy indicates not all of the elements in a compound are metal elements or silicon.

Table S2. Values of U-J Parameters Adopted in DFT+U Computations.

\begin{tabular}{ccccccccc}
\hline element & $\mathrm{V}$ & $\mathrm{Cr}$ & $\mathrm{Mn}$ & $\mathrm{Fe}$ & $\mathrm{Co}$ & $\mathrm{Ni}$ & $\mathrm{Mo}$ & $\mathrm{W}$ \\
\hline U-J & 3.25 & 3.70 & 3.90 & 5.30 & 3.32 & 6.20 & 4.38 & 6.2 \\
\hline
\end{tabular}



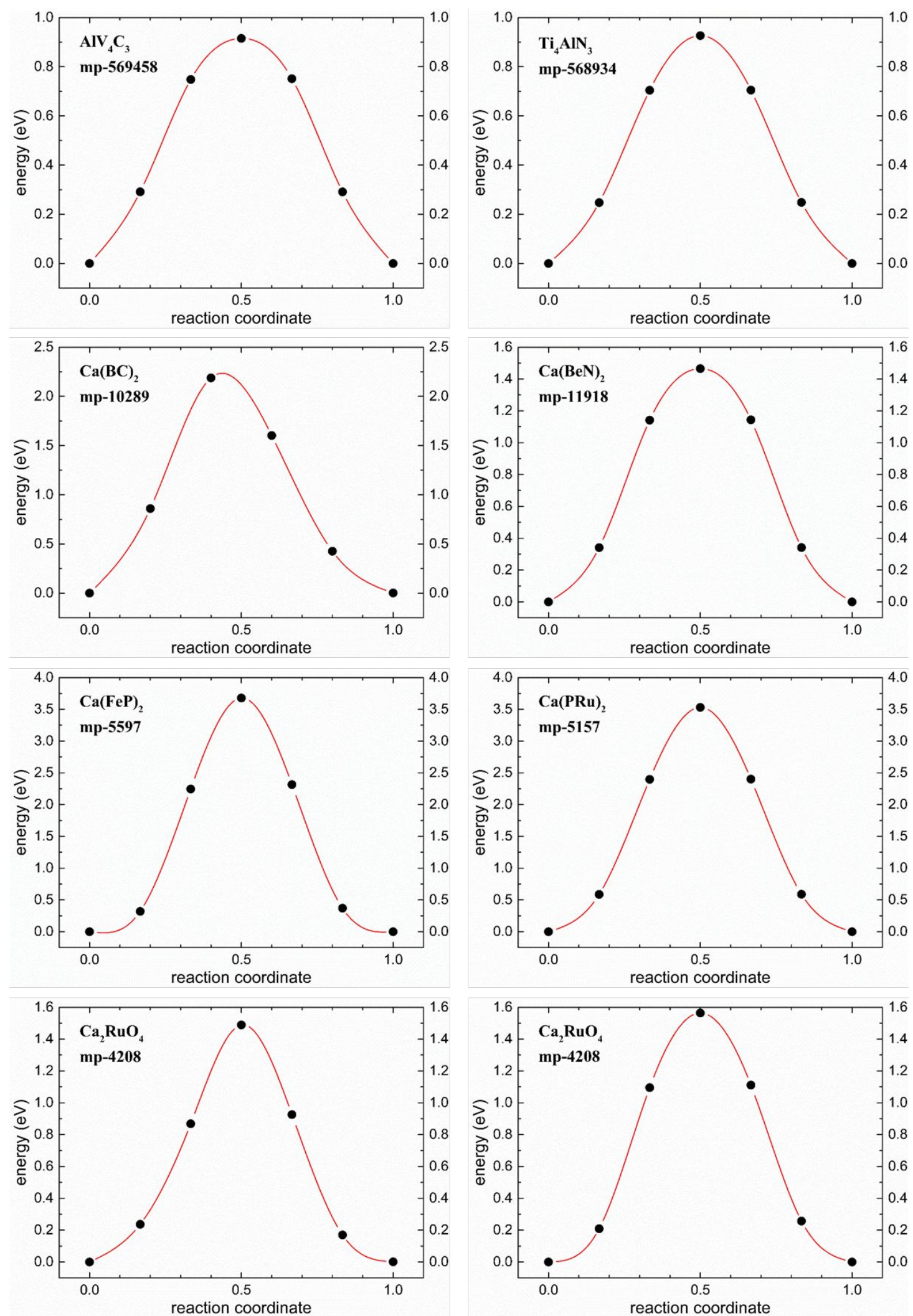

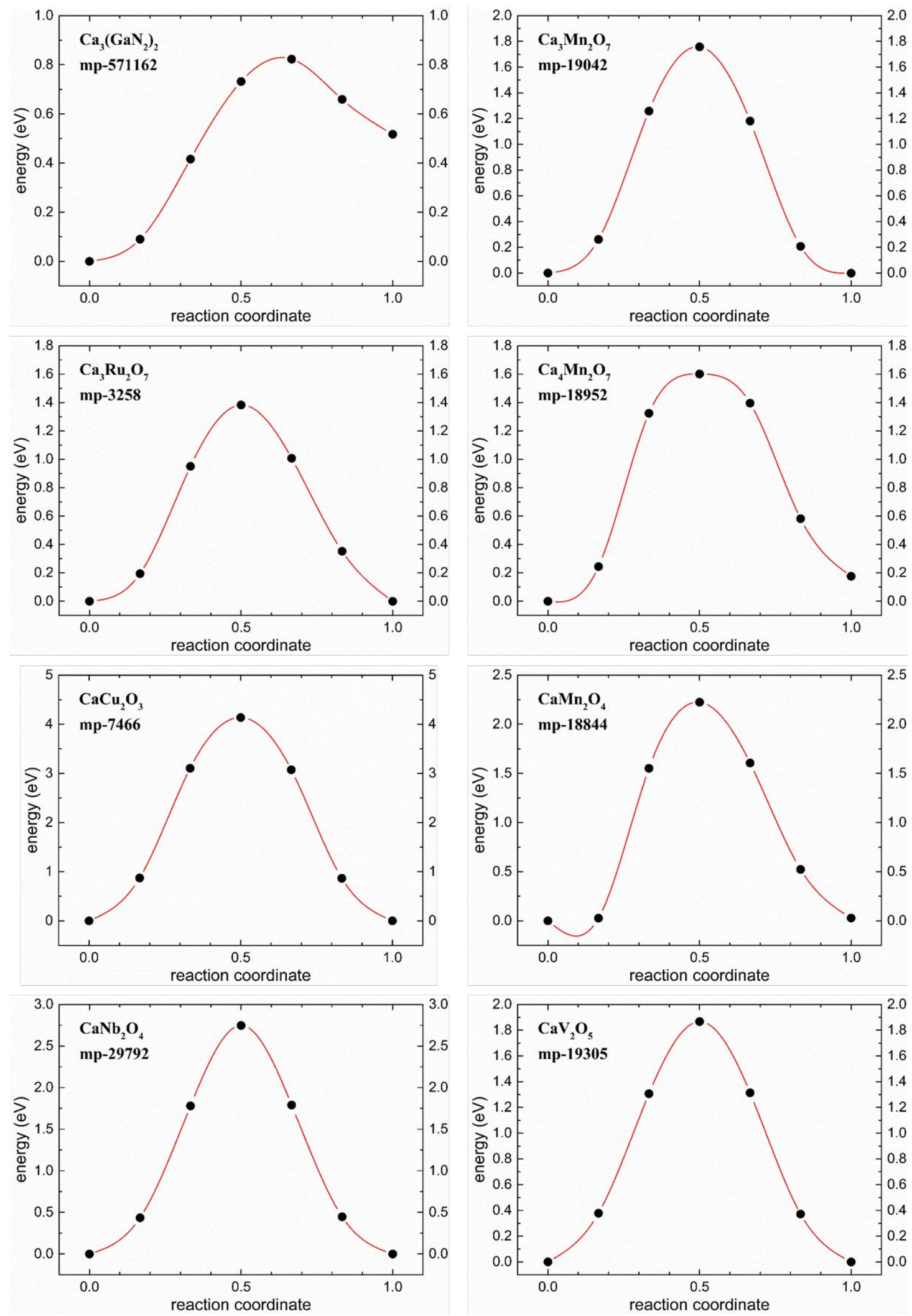

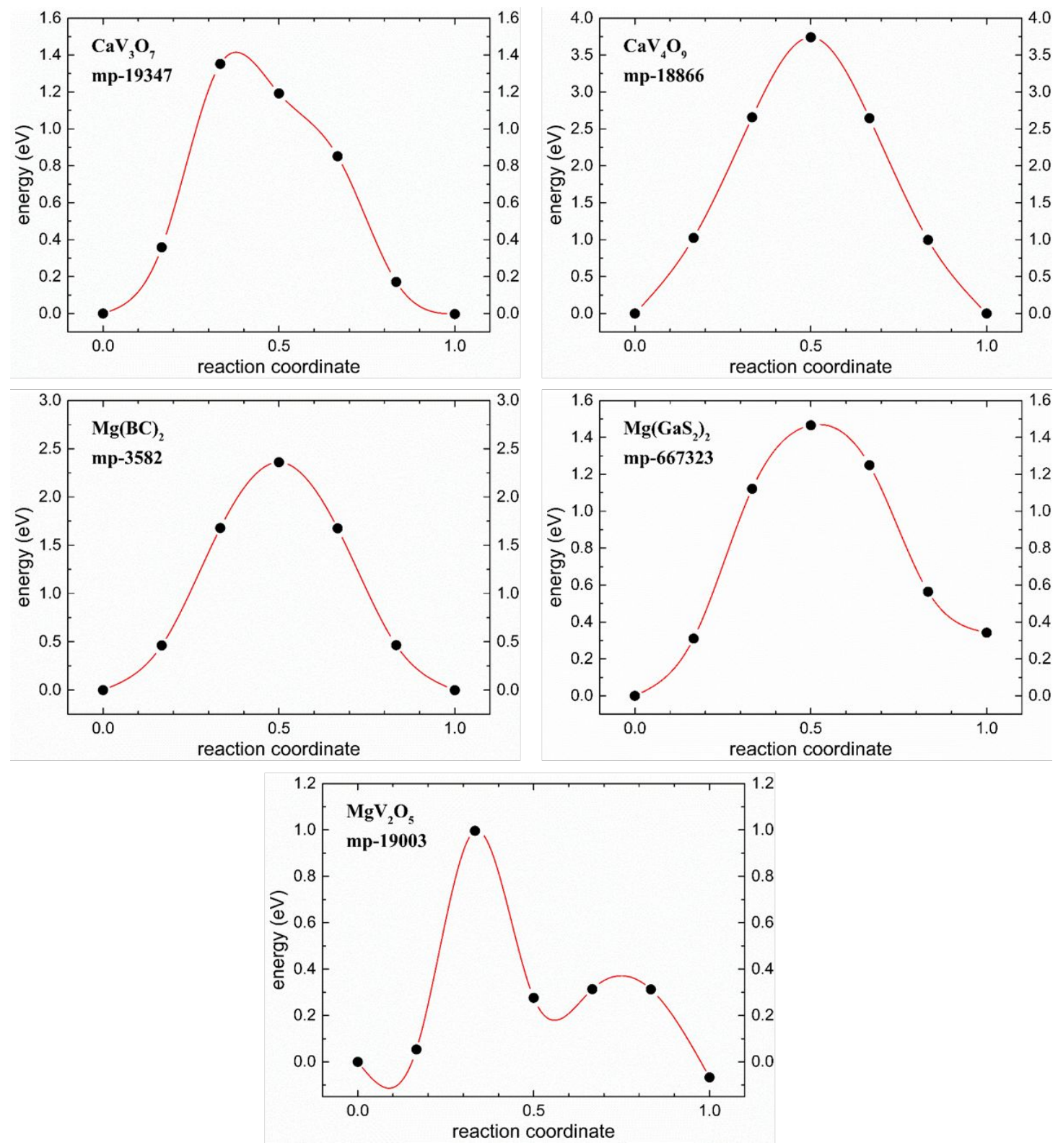

Figure S1. Energy profiles of multivalent ion diffusion in the obtained candidate cathode materials. 
Al
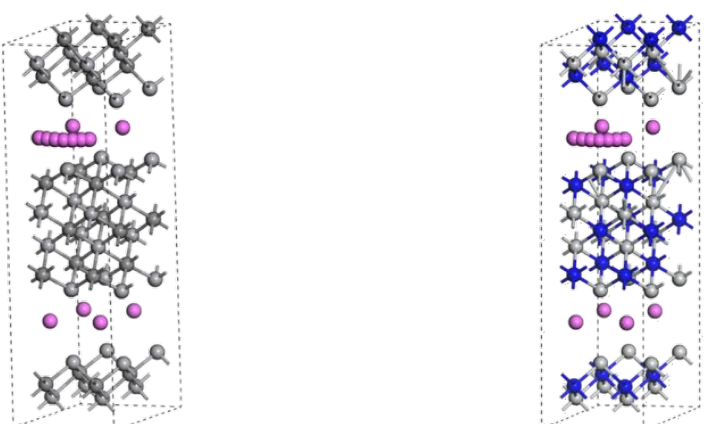

$$
\begin{gathered}
\mathrm{AlV}_{4} \mathrm{C}_{3} \\
\mathrm{mp}-569458
\end{gathered}
$$

$\mathrm{Ti}_{4} \mathrm{AlN}_{3}$ mp-568934

\section{Ca}
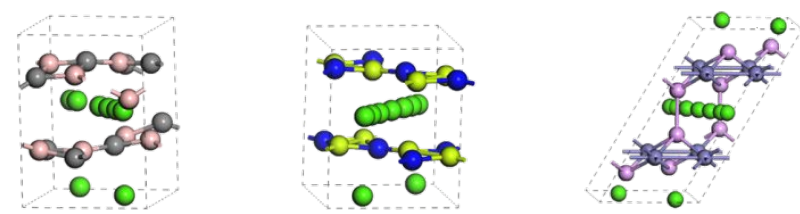

$\mathrm{Ca}(\mathrm{BC})_{2}$

mp-10289
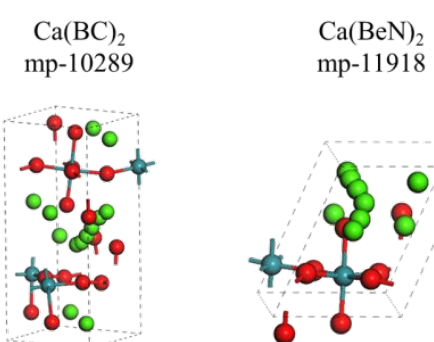

$\mathrm{Ca}_{2} \mathrm{RuO}_{4}$

mp-21466

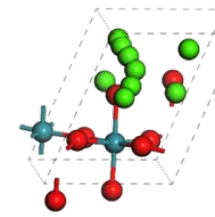

$\mathrm{Ca}_{2} \mathrm{RuO}_{4}$

mp-4208

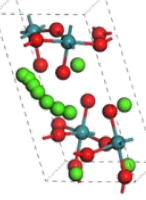

$\mathrm{Ca}_{3} \mathrm{Ru}_{2} \mathrm{O}_{7}$ mp-3258

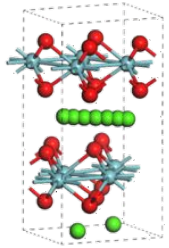

$\mathrm{CaNb}_{2} \mathrm{O}_{4}$ mp-29792

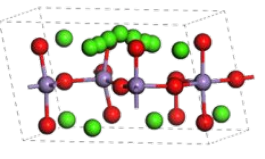

$\mathrm{Ca}_{4} \mathrm{Mn}_{2} \mathrm{O}_{7}$

$$
\text { mp-18952 }
$$

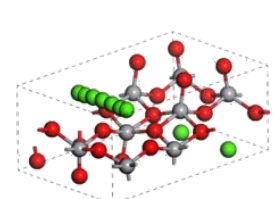

$\mathrm{CaV}_{2} \mathrm{O}_{5}$ mp-19305

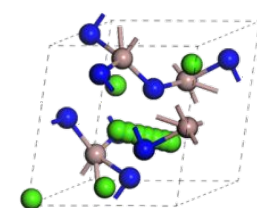

$\mathrm{Ca}_{3}\left(\mathrm{GaN}_{2}\right)_{2}$ mp-571162

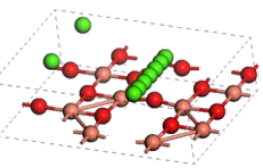

$\mathrm{CaCu}_{2} \mathrm{O}_{3}$ mp-7466

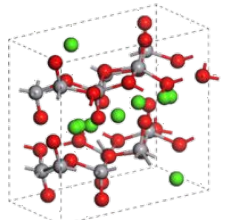

$\mathrm{CaV}_{3} \mathrm{O}_{7}$ mp-19347

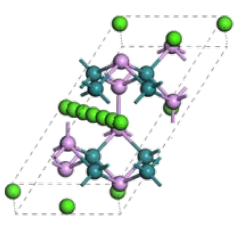

$\mathrm{Ca}(\mathrm{PRu})_{2}$ mp-5157

Mg
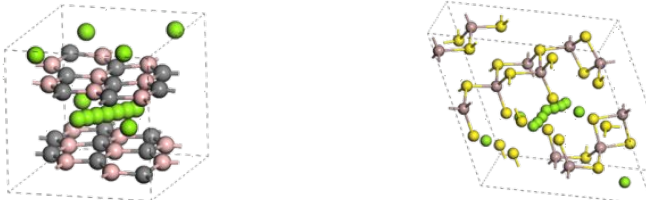

$\mathrm{Mg}(\mathrm{BC})_{2}$
$\mathrm{mp}-3582$

$\mathrm{Mg}\left(\mathrm{GaS}_{2}\right)_{2}$ mp-667323

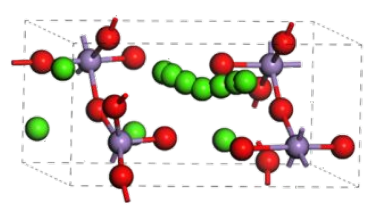

$\mathrm{Ca}_{3} \mathrm{Mn}_{2} \mathrm{O}_{7}$ mp-19042

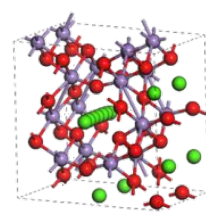

$\mathrm{CaMn}_{2} \mathrm{O}_{4}$ mp-18844

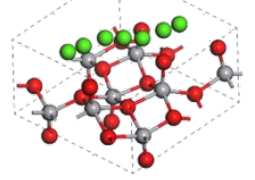

$\mathrm{CaV}_{4} \mathrm{O}_{9}$ mp-18866

Figure S2. Diffusion pathways of multivalent ions in the obtained candidate 
cathode materials. 


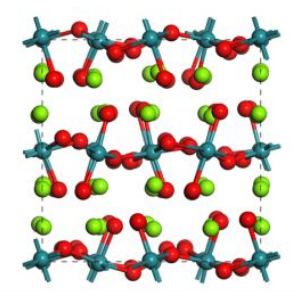

$\mathrm{Mg}_{2} \mathrm{RuO}_{4}$ PBCA

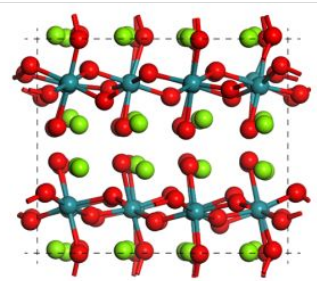

$\mathrm{Mg}_{3} \mathrm{Ru}_{2} \mathrm{O}_{7}$ CMC21

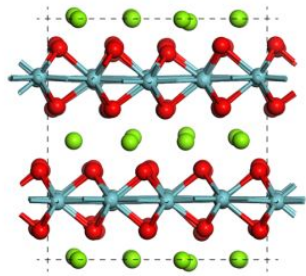

$\mathrm{MgNb}_{2} \mathrm{O}_{4}$ PCMB

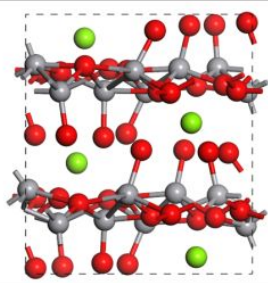

$\mathrm{MgV}_{4} \mathrm{O}_{9}$

$\mathrm{P} 4 / \mathrm{N}$

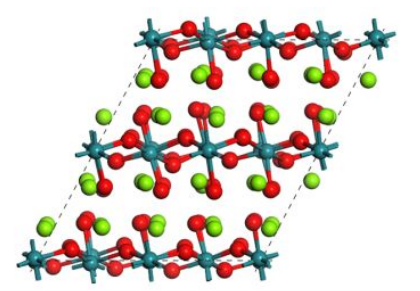

$\mathrm{Mg}_{2} \mathrm{RuO}_{4}$ BMAB

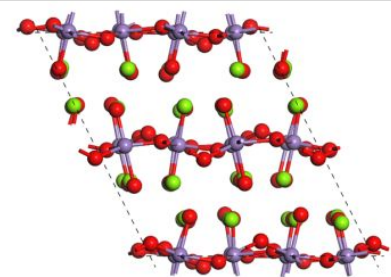

$\mathrm{Mg}_{4} \mathrm{Mn}_{2} \mathrm{O}_{7}$ $\mathrm{CMCA}$

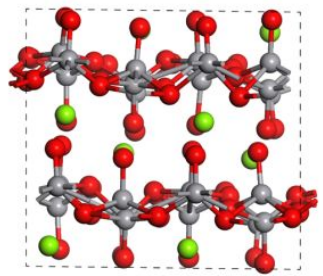

$\mathrm{MgV}_{3} \mathrm{O}_{7}$ P21

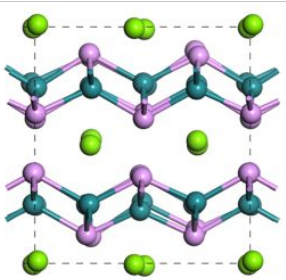

$\mathrm{Mg}(\mathrm{PRu})_{2}$

I4/MMM

Figure S3. Structures after $10 \mathrm{ps}$ AIMD at $600 \mathrm{~K}$ of the MIB cathode materials derived from the CIB cathode candidates. 

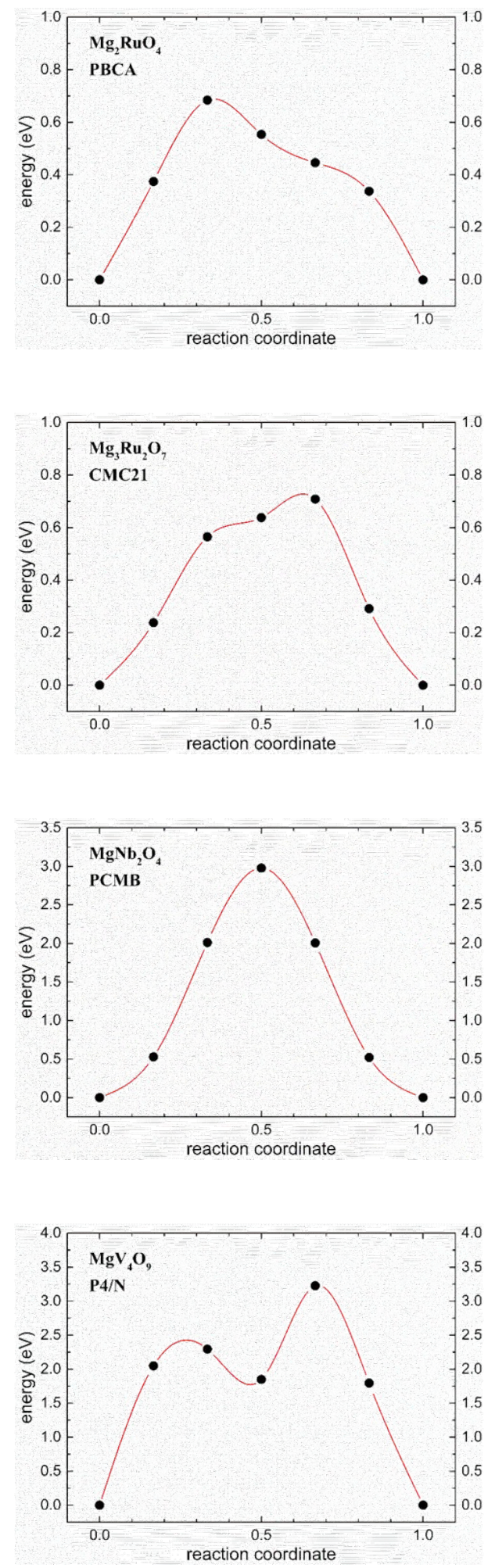
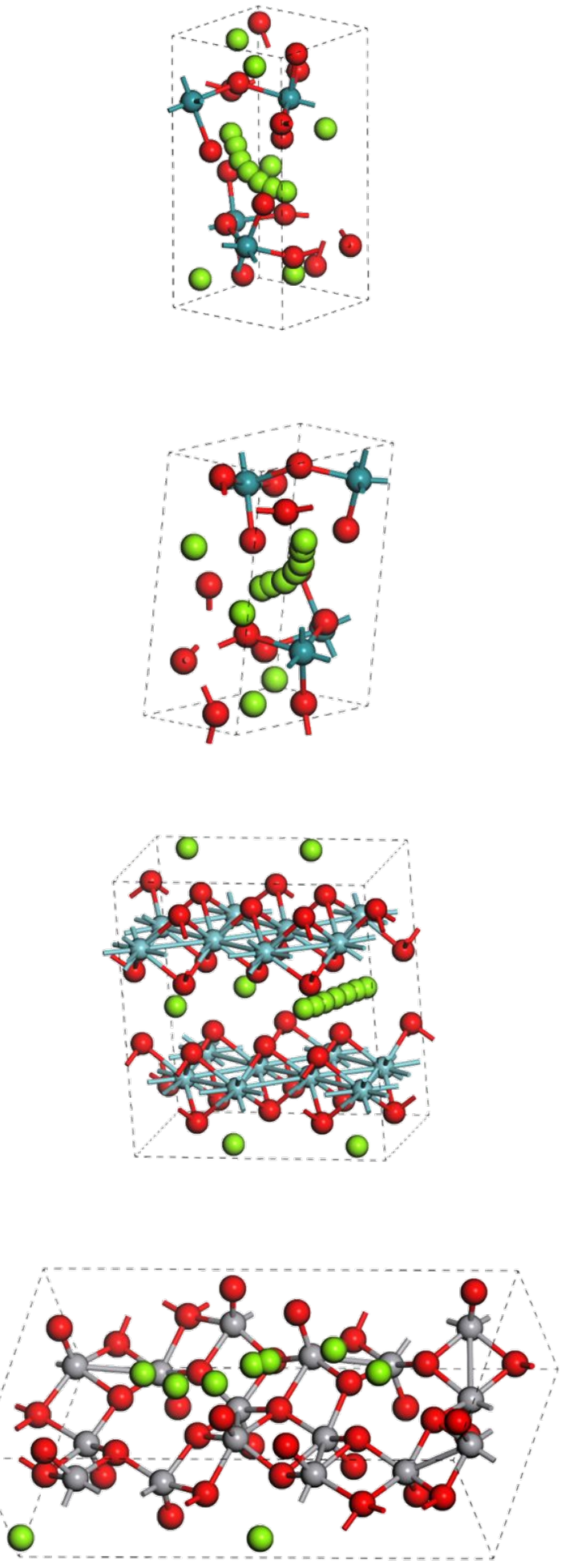

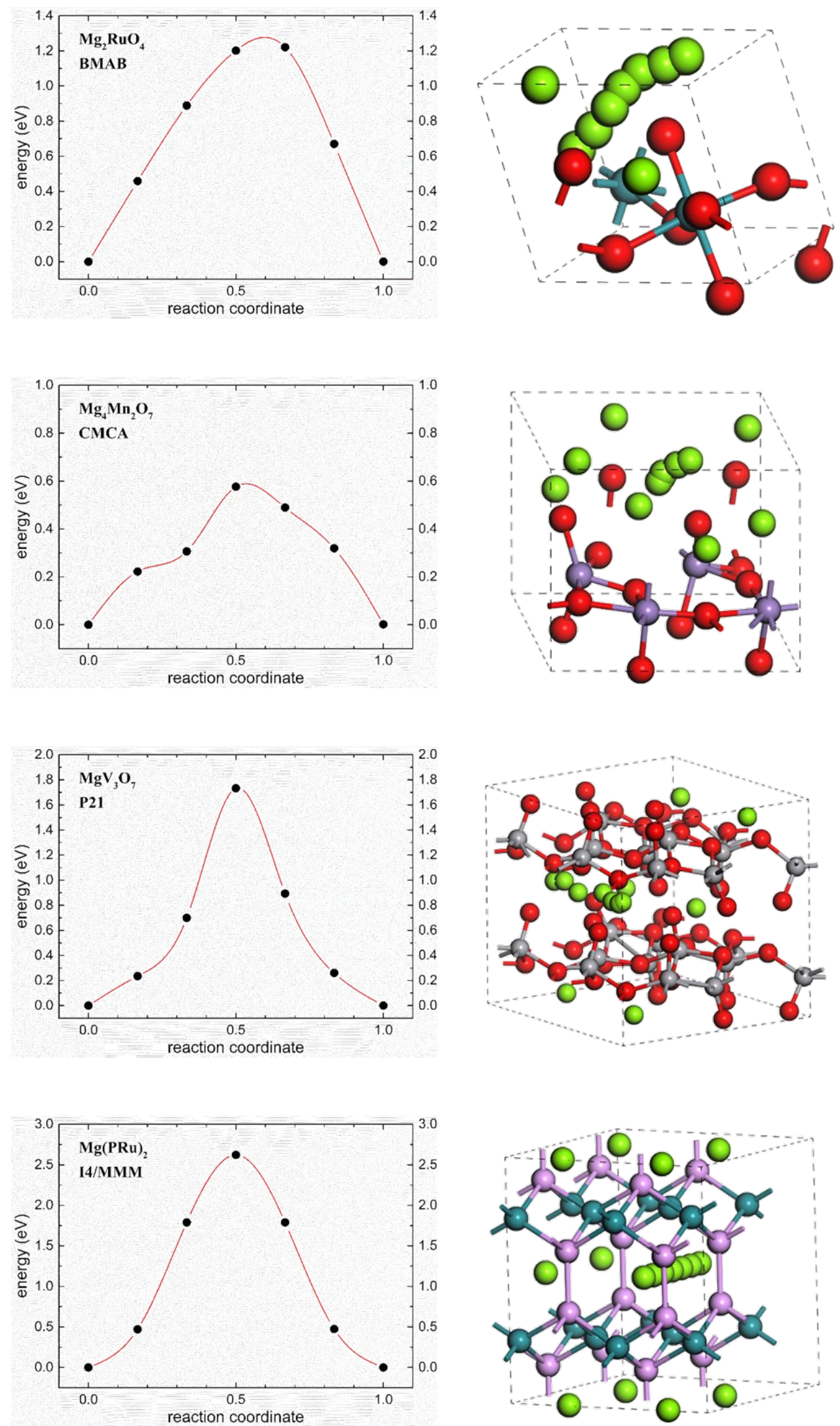

Figure S4. Energy profiles and diffusion pathways of multivalent ions in the Mg containing cathode candidates derived from their Ca containing counterparts. 
